\title{
BMJ Open Cholinesterase alterations in delirium after cardiosurgery: a German monocentric prospective study
}

\author{
Elisabeth Hannah Adam (D , , Victoria Haas, ${ }^{1}$ Simone Lindau, ${ }^{1}$ Kai Zacharowski, ${ }^{1}$ \\ Bertram Scheller ${ }^{2}$
}

To cite: Adam EH, Haas V, Lindau S, et al. Cholinesterase alterations in delirium after cardiosurgery: a German monocentric prospective study. BMJ Open 2020;10:e031212. doi:10.1136/ bmjopen-2019-031212

- Prepublication history for this paper is available online. To view these files please visit the journal online (http://dx.doi org/10.1136/bmjopen-2019031212).

Received 22 April 2019 Revised 09 December 2019 Accepted 10 December 2019

Check for updates

(c) Author(s) (or their employer(s)) 2020. Re-use permitted under CC BY-NC. No commercial re-use. See rights and permissions. Published by BMJ.

${ }^{1}$ Department of Anesthesiology, Intensive Care Medicine and

Pain Therapy, University Hospital Frankfurt, Frankfurt am Main, Germany

${ }^{2}$ Department of Anesthesiology, Intensive Care Medicine and Pain Therapy, Evangelisches Krankenhaus Düsseldorf, Dusseldorf, Germany

Correspondence to Dr Elisabeth Hannah Adam; elisabeth.adam@kgu.de

\section{ABSTRACT}

Objectives Postoperative delirium (POD) is a common complication after elective cardiac surgery. Recent evidence indicates that a disruption in the normal activity of the cholinergic system may be associated with delirium. Design Prospective observational study.

Setting Single-centre at a European academic hospital. Primary and secondary outcome measures In our study the enzyme activities of acetylcholinesterase (AChE) and butyrylcholinesterase (BChE) were determined preoperatively as well as on the first and second postoperative day. The confusion assessment method for the intensive care unit was used to screen patients for the presence of POD.

Results A total of 114 patients were included in the study. POD was associated with a decrease in BChE activity on postoperative day $1(p=0.03)$. In addition, patients who developed POD, had significantly lower preoperative AChE activity than patients without POD $(p<0.01)$. Multivariate analysis identified a preoperatively decreased AChE activity (OR 3.1; 95\% Cl 1.14 to 8.46), anticholinergic treatment (OR 5.09; $95 \% \mathrm{Cl} 1.51$ to 17.23 ), elevated European System for Cardiac Operative Risk Evaluation (OR 3.68; $95 \% \mathrm{Cl} 1.04$ to 12.99 ) and age (OR 3.02; $95 \% \mathrm{Cl} 1.06$ to 8.62 ) to be independently associated with the development of POD.

Conclusions We conclude that a reduction in the acetylcholine hydrolysing enzyme activity in patients undergoing cardiac surgery may correlate with the development of POD.

\section{INTRODUCTION}

Delirium is a complex neuropsychiatric syndrome that is clinically characterised by sudden onset and fluctuating course. Clinical symptoms according to the actual definition of the Diagnostic and Statistical Manual of the American Psychiatric Association (DSM-5 $)^{1}$ include disturbances in attention, awareness and another cognitive domain. Delirium is characterised as an aetiologically unspecific cerebro-organic syndrome representing a decompensation of cerebral function. ${ }^{2}$ The duration of delirium varies greatly and the severity ranges from mild to serious conditions.

\section{Strengths and limitations of this study}

One strength of this study results from the prospective nature.

Another strength is the data acquisition from a highvolume centre.

- A limitation is the inclusion limited to cardiac surgery patients as it remains unclear whether the results can be extrapolated to other patient cohorts.

- As the symptoms of delirium may vary over time, there may be a possibility that not all patients with delirium were detected, due to a single assessment of delirium per day.

The causes for delirium are multifactorial. Risk factors include dehydration, sleep deprivation, age, hypoxia, substance intoxication, anaemia and hypoglycaemia. In the general population, the incidence is below $0.4 \%$, in hospitalised patients between $15 \%$ and 22\%. ${ }^{34}$ Particularly after surgical interventions, patients are at risk of developing postoperative delirium (POD). The incidence is described to be as high as $52 \% .^{5}$ The consequences of a POD are very different and range from prolonged hospital stay, increased risk of wound infections, reduced quality of life, more frequent discharge into nursing homes to increased mortality in the first year after surgery. ${ }^{6-9}$ Recent literature suggests a significant association between frailty and the development of POD with an OR of almost 10, while limitations are considerable due to notable methodological heterogeneity between the methods of studies on such associations. ${ }^{10}$ Another risk factor for the development of POD may be a preoperative cognitive impairment, as observed in patients undergoing vascular surgery with a demonstrated OR of greater than 2. $^{11}$

Higher age, longer duration of surgery as well as a reduced preoperative cognitive condition are frequently found in cardiac surgery patients and increase the risk for 
development of POD in this group of patients. ${ }^{4}$ In the literature, the incidence of POD after cardiac surgery varies from $8 \%$ to $52 \% .^{45912}$ The duration of the POD in such patients varies widely, lasting 3 days on average. ${ }^{6}{ }^{13}$ Patients with POD are at risk for developing chronic postoperative cognitive dysfunction (POCD) over time and for suffering from severe long-term cognitive deficits. ${ }^{14}$

There are different hypotheses about the molecular mechanisms involved in the development of delirium. ${ }^{15}$ The most common hypothesis for the development of POD is based on a central cholinergic deficit resulting from a deficit of acetylcholine (ACh): pathologies at the presynapse, in the synaptic cleft or at the postsynaptic receptor may trigger a central cholinergic deficit. Acetylcholinesterase (AChE) is an enzyme which cleaves ACh in the synaptic cleft and terminates the transmission of a stimulus, a prerequisite for generating a new impulse. If the AChE is restricted in its function ACh remains in the synaptic cleft and blocks a new stimulus transmission. ${ }^{16}$ However, several authors have found data challenging this hypothesis as they did not identify an association of preoperative serum anticholinergic activity with the development of $\mathrm{POD}^{17}$ or a therapeutic effect of rivastigmine for the prevention of POD. ${ }^{18}$ Other hypotheses (eg, brain injury, metabolic abnormalities) are based on localised or general brain energy deprivation critical to attentional processes such as the caudate nucleus or frontal cholinergic pathways. ${ }^{17}$ Systemic inflammation may cause alterations including proinflammatory cytokines and prostaglandins mediated by humoral and neural signalling pathways leading to symptoms of delirium. ${ }^{18}$

Butyrylcholinesterase (BChE) is an enzyme which splits choline compounds as well as other esters. ${ }^{19}$ For a long time $\mathrm{BChE}$ was thought to have a less important function, but recent literature demonstrated that BChE may in part and with a significantly slower rate and affinity act as a substitute in the absence of AChE with a relevant role in the development of a cholinergic deficit. ${ }^{20} 21$

A recently published study identified a significant decrease in the enzyme activity of AChE and BChE in patients with POD after hip surgery. ${ }^{22}$ However, the impact of a choline esterase deficit in patients remains unclear. Previously published manuscripts on the impact of cholinesterase activity on POD in surgical patients reach different conclusions. While postoperative measurement of AChE and BChE did not discern between patients with and without POD in a study published by John et $a l$, Müller et al found a potential relationship between cholinesterase activity and the development of POD. ${ }^{23} 24$ While John et al only studied postoperative cholinesterase activity, we sought to incorporate preoperative cholinesterase activity in order to assess a potential implication on the development of POD. In contrast to the study of Müller et al we only included patients undergoing cardiac surgery in order to decrease heterogeneity.

Due to the far-reaching consequences of a POD, it is of great importance to identify patients at risk for the development of such a disorder. Our study investigated the extent to which changes in bed-side enzyme activity of cholinesterases correlates with the development of POD in cardiac surgery patients and to identify possible factors influencing the development of POD.

\section{MATERIAL AND METHODS}

This manuscript includes data gained during a prospective observational study at the University Hospital Frankfurt.

\section{Patient and public involvement}

Patients were included between February 2013 and February 2014. Over this period, 150 patients who received elective cardiac surgery at the University Hospital Frankfurt were screened for inclusion. The participating patients were informed about the study verbally and in writing. Only patients with written consent were included in the study.

Potential patients had to meet the following inclusion criteria: elective cardiac surgery with and without the use of a cardiopulmonary bypass (CPB) and age over 18 years. Exclusion from the study was based on: preoperatively existing delirium; preoperatively sedated patients with Richmond Agitation and Sedation Scale (RASS) $<-2$; no proficiency of the German or English language or missing patient consent.

\section{Design}

After obtaining consent, patients were examined preoperatively and on the first and second postoperative day. Patients were examined for the presence of a POD using the confusion assessment method for the intensive care unit (CAM-ICU) clinical test. ${ }^{25}$ In brief, the CAMICU assesses and scores clinical features associated with delirium. Depending on the results from CAM-ICU, a patient was assigned to either the POD group or the no POD group. The patient was assigned to the POD group if delirium was diagnosed at least once as per the CAMICU. If a patient was either under too much sedation or the examiner was not able to apply the CAM-ICU, the patient was not included for analysis.

\section{Assessment of parameters}

All included patients were scheduled for elective surgery and assessed directly before surgery at 07:00 am to determine the presence of delirium. First, the RASS score was obtained, then blood samples were taken for the assessment of BChE and AChE activity. Further, blood samples were analysed for AChE and BChE activity as measured with the ChE Check Mobile (Securetec DetektionsSysteme AG, Neubiberg, Germany). Both, BChE and AChE activity were assessed using the ChE Check Mobile as per the manufacturer's instruction. Preoperatively, blood samples were drawn from the fingertip $(10 \mu \mathrm{L})$. Postoperatively, blood samples $(1 \mathrm{~mL})$ were obtained via an arterial line. As two enzymes were determined in 
different measurements, two blood samples were taken at different times and analysed independently. To provide consistency between assessments, measurement of AChE activity was always performed first, followed by assessment of BChE activity. Measurements were about $10 \mathrm{~min}$ apart. As animal data on the circadian changes of cholinesterase reveal a relevant increase during the sleep phase, we have hence taken samples at the same time preoperatively ( \pm 1 hour) to ensure consistency of measurements. ${ }^{26}$

The ChE Check Mobile device incorporates a variety of factors contributing to a more precise analysis of cholinesterase activity. ${ }^{27}$ Working conditions and technical data for this device are published online. ${ }^{28}$ Previously, detailed information on the accuracy of this device have been published before having demonstrated acceptable reliability for the measurement of cholinesterases. ${ }^{29}$ Further, this device has been used in the context of POD before. $^{2324}$

\section{Data collection}

Basic demographic data, medication, hospitalisation period, the length of stay on the ICU, ventilation time as well as postoperative medication, transfusion, information about secondary diagnoses, weight, laboratory values as well as obtained scores were extracted from the patient data management system. Further, the European System for Cardiac Operative Risk Evaluation (EuroSCORE) ${ }^{30}$ was calculated for each patient. The EuroSCORE is a risk model that facilitates a calculation of the risk of death after heart surgery. The model asks for 17 parameters about the patient, the condition of the heart and the proposed surgery and calculates the risk of death. The EuroSCORE has become the most widely used risk index for cardiac surgery, potentially improving the results of cardiac surgery. Medication was considered to be anticholinergic based on the study by Ancelin et al. ${ }^{31}$ The duration of anaesthesia, intraoperative medication, aortic clamping time and the duration of CPB were extracted from the anaesthesia and premedication protocols. The data and results were inserted and maintained in an Excel database.

\section{Statistics}

All data were tested for normality using the D'Agostino and Pearson omnibus normality test. Data comparisons of patient characteristics were made using Mann-Whitney $\mathrm{U}$ test or $\chi^{2}$-test, where applicable. To compare activities of cholinesterases between different days, a Wilcoxon signedrank test was used. Univariate analysis was performed using the $\chi^{2}$-test. Non-binary-parameters were stratified by the median. Parameters with a $\mathrm{p}$ value less than 0.1 were included for multivariate analysis, as carried out by binary logistic regression.

Length of ventilation was defined as the time of intubation until extubation; length of stay on the ICU was defined as the time from surgery to the discharge from the postoperative ICU; length of stay in the hospital was defined as the time from surgery to discharge from the primary care hospital. For survival analysis, groups were compared using a log rank test and pointwise $95 \%$ CIs. A multivariate Cox's proportional hazards regression backward stepwise model (likelihood ratio) was performed to find independent predictors for outcome parameters.

Results with $\mathrm{p}<0.05$ were considered to be statistically significant. All calculations/analyses were performed with SPSS (V.25) or Graphpad Prism (V.5.0, La Jolla, California, USA). No correction for multiple comparisons were performed for secondary outcome analysis. Hence, results on secondary outcomes are to be considered exploratory. ${ }^{32}$

\section{RESULTS}

\section{Participants}

Of the 150 patients screened for this study, 13 were excluded due to cancelled surgery and 23 were excluded due to an unavailability for assessment of delirium resulting from prolonged sedation thus leaving 114 patients available for analysis. Of the 114 patients included within our study, 31 patients $(27.2 \%)$ developed a POD, while 83 patients $(72.8 \%)$ did not show signs of a POD.

\section{Baseline characteristics}

No statistical differences were observed for sex, body mass index, in-hospital death, preoperative incidence of alcohol abuse, the preoperative prescription of anticholinergic drugs or the performed procedure (table 1). Of note, none of the patients without history of anticholinergic medication received anticholinergic medication throughout the ICU stay. However, patients who went on to develop a POD had a significantly higher EuroSCORE $(p=0.02)$. Further, patients who developed POD were significantly older than patients without the development of POD $(\mathrm{p}<0.01)$.

\section{Outcome dependent on the development of POD}

Patients without the development of POD displayed a significantly shorter length of ventilation $(\mathrm{p}=0.02)$, shorter length of stay in the ICU $(\mathrm{p}<0.01)$ and shorter length of hospitalisation $(\mathrm{p}<0.01)$ (table 1$)$. No differences were observed in regard to mortality, when comparing patients with or without the development of POD.

\section{Assessment of cholinesterases}

In the overall study population, the BChE decreased significantly over time, when comparing mean $\mathrm{BChE}$ activity on postoperative days $1(\mathrm{p}<0.01)$ and $2(\mathrm{p}<0.01)$ with the preoperative BChE activity (figure $1 \mathrm{~A}$ ). Further, the mean AChE activity increased over time, when comparing the AChE activity on postoperative day 2 with the preoperative AChE activity $(\mathrm{p}=0.03$ ) (figure $1 \mathrm{~B}$ ).

No significant preoperative difference in $\mathrm{BChE}$ activity was observed in patients with or without POD (figure 2A). Significant differences were observed in regard to the activity of $\mathrm{BChE}$ on postoperative day $1 \quad(\mathrm{p}=0.03)$ (figure 2B), when comparing patients from the POD 
Table 1 Patient characteristics

\begin{tabular}{|c|c|c|c|}
\hline & $\begin{array}{l}\text { No postoperative delirium } \\
(n=83)\end{array}$ & $\begin{array}{l}\text { Postoperative delirium } \\
(\mathrm{n}=31)\end{array}$ & \\
\hline Age (years (IQR)) & $69(58-74)$ & $74(71-78)$ & $<0.01^{*}$ \\
\hline Female sex (n (\%)) & $22(26.5)$ & $9(29)$ & 0.79 \\
\hline EuroSCORE (n (\%)) & & & 0.02 \\
\hline $1-5$ & $59(71.1)$ & $13(41.9)$ & \\
\hline $6-10$ & $22(26.5)$ & $16(51.6)$ & \\
\hline $11-15$ & $2(2.4)$ & $2(6.5)$ & \\
\hline Body mass index $\left(\mathrm{kg} / \mathrm{m}^{2}(\mathrm{SD})\right)$ & $27.6( \pm 4.8)$ & $28(4.8)$ & $0.7^{\star}$ \\
\hline Alcohol abuse (n (\%)) & $2(2.4)$ & 0 & 1 \\
\hline Anticholinergic premedication (n (\%)) & $8(9.9)$ & $10(32.3)$ & $<0.01$ \\
\hline Procedure (n (\%)) & & & 0.3 \\
\hline CABG & $33(39.8)$ & $15(48.4)$ & \\
\hline AVR & $24(28.9)$ & $6(19.4)$ & \\
\hline Combined procedure & $10(12)$ & $6(19.4)$ & \\
\hline TAVI & $4(4.9)$ & $3(9.7)$ & \\
\hline MVR & $6(7.2)$ & $1(3.1)$ & \\
\hline Other & $6(7.2)$ & 0 & \\
\hline Length of ventilation ( $\min (\mathrm{SD}))$ & $471( \pm 159)$ & $1427( \pm 3565)$ & $0.02^{*}$ \\
\hline Length of stay on ICU (hours (SD)) & $20.1( \pm 20.1)$ & $93.5( \pm 183)$ & $<0.01^{*}$ \\
\hline Length of stay in hospital (days (SD)) & $13.1( \pm 5)$ & $20.9(13.9)$ & $<0.01^{*}$ \\
\hline In-hospital death (n (\%)) & $1(1.2)$ & $1(3.2)$ & $0.47^{*}$ \\
\hline Preop BChE activity (U/g Hb (median, SD)) & $2773(2740 \pm 885)$ & $2734(2891 \pm 922)$ & 0.83 \\
\hline PO day 1 BChE activity (U/g Hb (median, SD)) & $1966(1971 \pm 588)$ & $1674(1752 \pm 730)$ & 0.03 \\
\hline PO day 2 BChE activity (U/g Hb (median, SD)) & $1870(1868 \pm 564)$ & $1694(1715 \pm 596)$ & 0.16 \\
\hline Preop AChE activity (U/g Hb (median, SD)) & $45.4(45 \pm 5.7)$ & $42.2(41.5 \pm 6.3)$ & $<0.01^{*}$ \\
\hline PO day 1 AChE activity (U/g Hb (median, SD)) & $45.1(44.1 \pm 5.1)$ & $41.8(42 \pm 5.5)$ & $<0.01^{*}$ \\
\hline PO day 2 AChE activity (U/g Hb (median, SD)) & $45.5(45.6 \pm 4.6)$ & $42.7(42.8 \pm 5.8)$ & $<0.01^{*}$ \\
\hline
\end{tabular}

Data are given as means except for age which is presented as the median and as indicated. Data comparisons were made with the $t-$ test or the $\chi^{2}$-test, where applicable.

*Use of a non-parametric test due to non-normal distribution of data.

AChE, acetylcholinesterase; AVR, aortic valve replacement; BChE, butyrylcholinesterase; CABG, coronary artery bypass grafting; EuroSCORE, European System for Cardiac Operative Risk Evaluation; ICU, intensive care unit; MVR, mitral valve replacement; PO, postoperative; TAVI, transcatheter aortic valve replacement.

and the no-POD groups. However, no significant difference in BChE activity was observed on postoperative day 2 (figure 2C) between patients with or without POD. Further, patients with the development of POD displayed significantly lower levels of AChE activity preoperatively $(\mathrm{p}<0.01)$ and on postoperative days $1 \quad(\mathrm{p}<0.01)$ and 2 $(\mathrm{p}<0.01)$ (figure 2D-F).

\section{Parameters associated with POD}

To identify parameters associated with the development of POD in patients undergoing cardiac surgery, we performed a univariate analysis and identified age $>71$ years, EuroSCORE $\geq 4$, anticholinergic premedication and a preoperative AChE activity of $<44.3 \mathrm{U} / \mathrm{g} \mathrm{Hb}$ (table 2). To rule out potential confounding variables we performed a multivariate analysis and confirmed age $>71$ years, EuroSCORE $\geq 4$, preoperative anticholinergic medication and preoperative AChE activity of $<44.3 \mathrm{U} / \mathrm{g}$ $\mathrm{Hb}$ as parameters independently associated with the development of POD.

\section{Parameters associated with length of stay on the ICU}

Survival analysis demonstrated that patients with POD after cardiothoracic surgery displayed significantly longer length of stay in the ICU (figure 3). To identify further parameters associated with prolonged stay in the ICU following cardiothoracic surgery, we performed various univariate analyses and identified EuroSCORE $\geq 4$, preoperative anticholinergic medication, length of ventilation, transfusion of packed red blood cells, reduced AChE activity on postoperative day 1 , reduced postoperative BChE activity on postoperative day 1 and the development of POD as potentially associated (table 3 ). To identify confounders, we performed a multivariate analysis and 
A

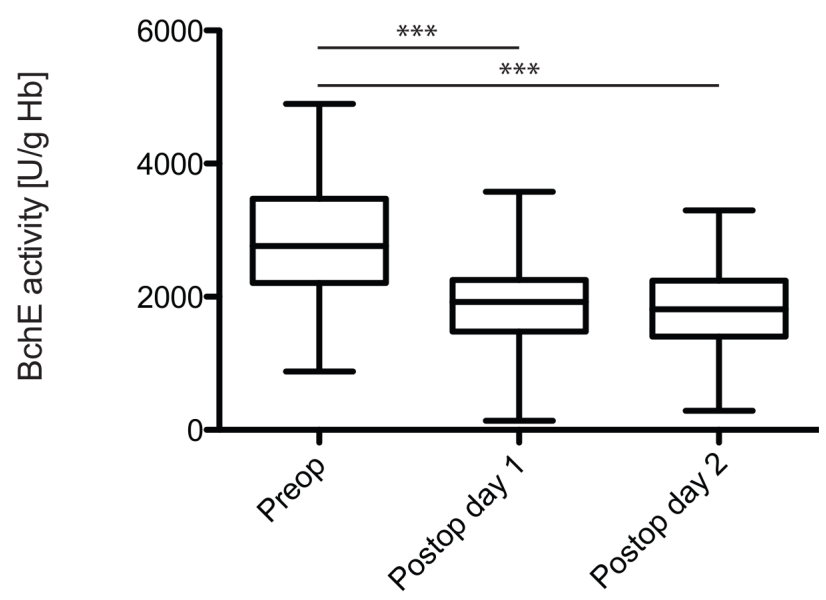

B

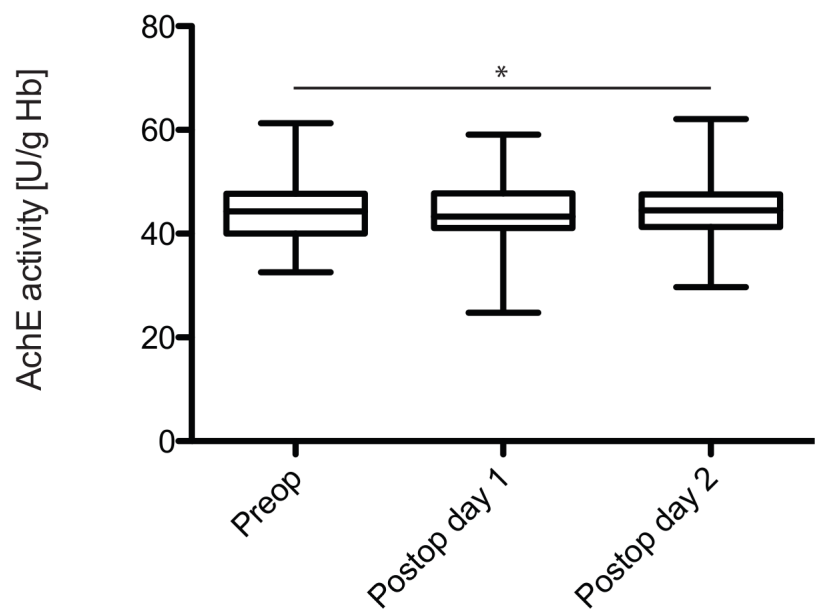

Figure 1 Activity of butyrylcholinesterase (BChE) and acetylcholinesterase (AChE) in the overall patient population. Activity of (A) BChE and (B) AChE were assessed preoperatively and on postoperative days 1 and $2 .{ }^{* \star} P$ value of $<0.01$; ${ }^{*} p$ value of 0.04 .

identified length of ventilation, reduced $\mathrm{BChE}$ activity on postoperative day 1 and the development of POD as independently associated with prolonged length of stay in the ICU.

\section{DISCUSSION}

The purpose of this study was to analyse a potential correlation between AChE and BChE activities and the incidence of POD in cardiosurgical patients and to identify further possible predictors for the development of POD.

The incidence of POD in our study population is in line with the literature. ${ }^{34}$ Our results show that a preoperative AChE activity was significantly lower in patients who went on to develop POD than in patients without the development of POD. Further, BChE activity was significantly lower in patients with POD on the first postoperative day. Our data revealed that the patients who developed a POD were significantly older than those who did not suffer from a POD. These patients were more frequently on anticholinergic medication. Further, the EuroSCORE was higher in such patients and they were longer ventilated. In addition, patients with POD stayed significantly longer in the ICU and were discharged significantly later for follow-up treatment.

Patients who went on to develop POD showed lower preoperative AChE activity compared with patients without the development of POD. This finding is in agreement with the current hypothesis that a reduction in AChE activity is associated with POD. It is hypothesised that due to this deficit, cholinesterase cannot efficiently cleave the neurotransmitter ACh in the synaptic cleft. As a consequence, the stimulus transmission cannot be terminated, and ultimately a new stimulus transmission cannot be initiated. ${ }^{33}$

In a recently published study, Cerejeira et al measured AChE and BChE activities preoperatively and postoperatively in patients who had undergone elective hip surgery and examined patients for the development of a POD using CAM-ICU. ${ }^{22}$ They came to the conclusion that patients with POD after surgery showed reduced preoperative AChE activity. As in our results, preoperative BChE activity was decreased in patients with POD. Contrary to their findings however, in our patient population groups with or without the development of POD did not differ significantly in preoperative BChE activity. This discrepancy might be attributed to different assays measuring enzyme activities. Most importantly, these findings need to be discussed in light of the 2017 publication by John et al. ${ }^{23}$ This group did not find any differences regarding both AChE and BChE activity between patients with or without the development of POD. However, there are some considerable differences in the study design: no preoperative samples were collected in the study by John et al. Further, some samples were refrigerated before analysis, thereby potentially altering the measured enzyme activity. Zivkovic et al, however, have also identified a reduced $\mathrm{BChE}$ activity following surgery. ${ }^{34}$ They suggested a cholinergic modulation of the inflammatory response that is independent of POD. This finding of a postoperatively decreased BChE activity and a potential association with POD as observed within our study needs to be addressed in further studies specifying the potential impact of cholinesterases in the development of POD, also in the context of inflammation. In a recently published manuscript, Müller et al found that perioperative peripheral cholinesterase activities may be related to the development of POD. ${ }^{24}$ In this study, cholinesterase activities were measured in surgical patients of various specialties. However, the authors of the above-named study stated the lack of a subgroup analysis discriminating between surgical procedures as a limitation of their study. In our study comprised patients undergoing cardiac surgery, we were able to find comparable results, potentially indicating an importance of cholinesterase activity in the development of POD. 
A

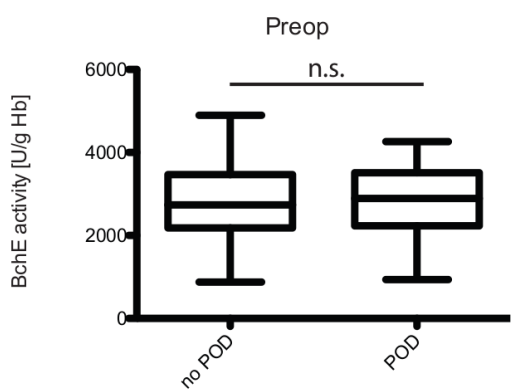

D

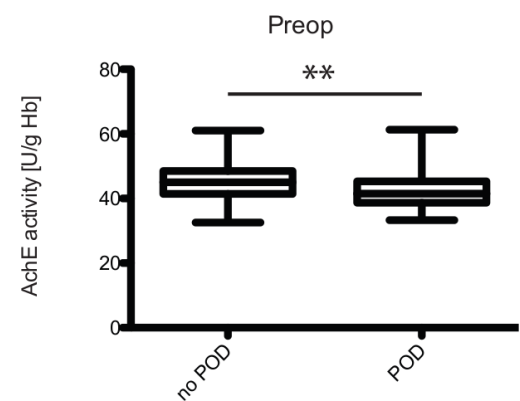

B

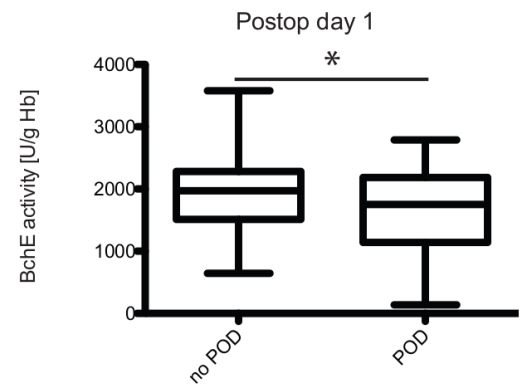

$\mathrm{E}$

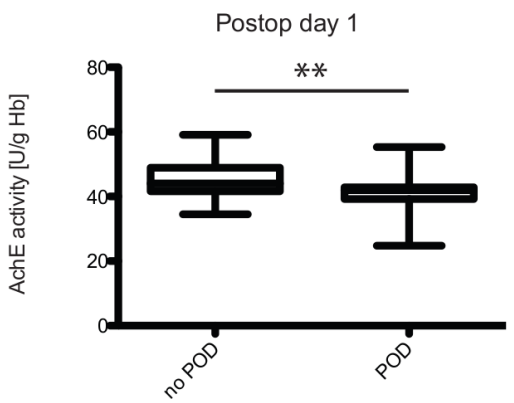

C

Figure 2 Activity of butyrylcholinesterase (BChE) and acetylcholinesterase (AChE) in patients without or with the development of postoperative delirium (POD). Activity of BChE was assessed (A) preoperatively and on postoperative days (B) 1 ( $^{*} \mathrm{p}$ value of 0.03 ) and (C) 2. Activity of AChE were assessed (D) preoperatively and on postoperative days (E) 1 and $(\mathrm{F}) 2 .{ }^{* \star} \mathrm{P}$ value of $<0.01$.

In a study conducted in 2008, Hubbard $e t$ al were able to show that a higher age was associated with deficits in the anticholinergic system. ${ }^{35}$ Photometric determination of AChE revealed no significant difference for BChE activity between younger and older age, but a significantly lower activity of cholinesterases in the older people displaying a significant amount of frailty. They suspected that age was associated with changes in enzyme activity. While a deficit in cholinesterase activity may be observed in elderly patients, a significant correlation with age could not be demonstrated. ${ }^{36-38}$ The association between age and the development of POD observed for our patient population fits well with the literature that described such association before ${ }^{39}$ In our cohort, patients with a history of

Table 2 Univariate and multivariate analysis of parameters associated with the development of postoperative delirium

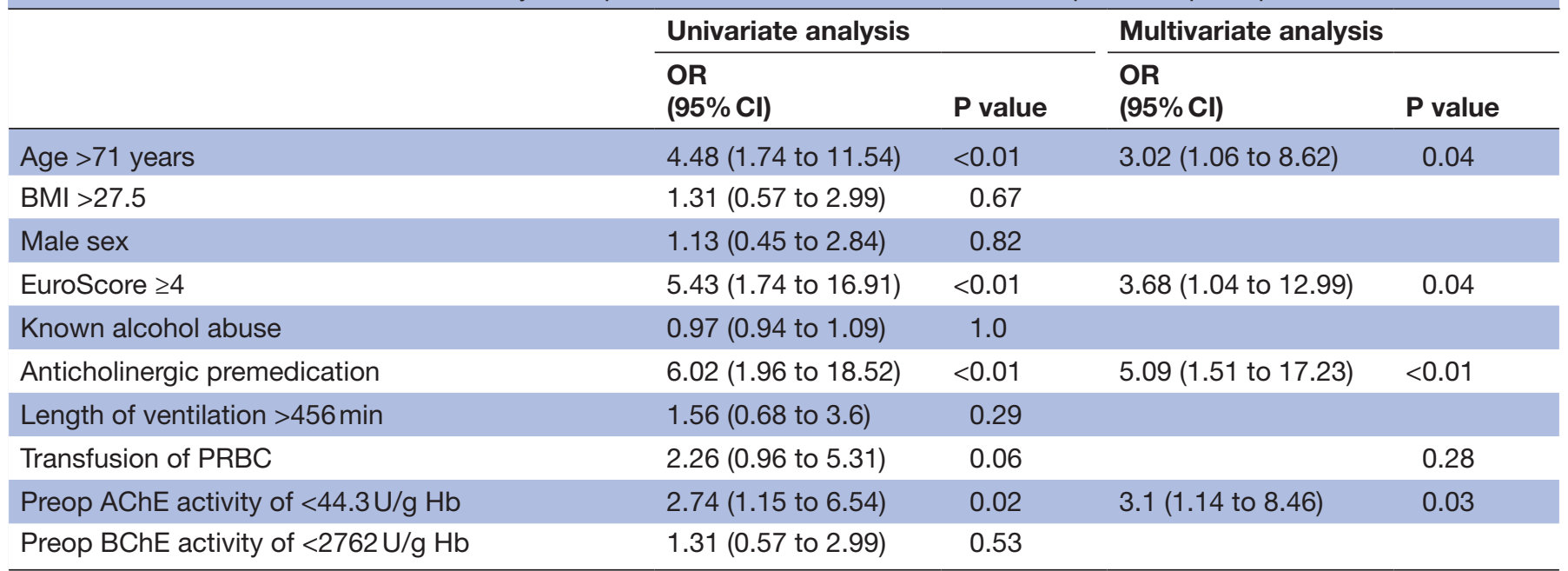

Data comparisons were made with $\chi^{2}$-test for univariate analysis, binary logistic regression with stepwise exclusion was used for multivariate analysis. For multivariate analysis $\mathrm{OR}$ is only displayed in significant outcome parameters/where applicable.

AChE, acetylcholinesterase; BChE, butyrylcholinesterase; BMI, body mass index; EuroSCORE, European System for Cardiac Operative Risk Evaluation; PRBC, packed red blood cells. 


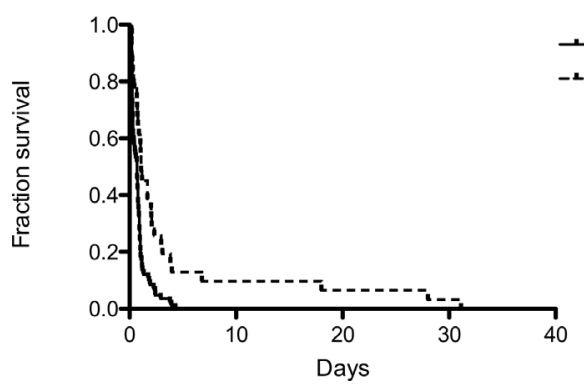

- No postoperative delirium -1- postoperative delirium

Figure 3 Kaplan-Meier estimate. Time to discharge from intensive care unit (log rank test $\chi^{2}=14.88, p<0.01$ ).

anticholinergic medication suffered from a POD significantly more often than patients in the comparison group. This result supports the assumption that the anticholinergic predisposition has an influence on the development of the POD. It reduces the function of ACh and might also related to a cholinergic deficit. Anticholinergic medication is used when patients are regularly treated with antidepressants (eg, amitriptyline, doxepin), anticonvulsants (eg, gabapentin) or for Parkinson's disease (benserazide, L-DOPA). These drugs all have in common that they reduce ACh activity through direct and indirect anticholinergic action. In a study conducted in 2016, Naja $e t$ al investigated geriatric patients with regard to the treatment with anticholinergic drugs before and during hospitalisation and the incidence of delirium. They came to the conclusion that the anticholinergic burden was associated with the occurrence of delirium and that anticholinergic exposure correlated with the incidence of delirium and increased mortality. ${ }^{40}$

When interpreting effect sizes of the above-named potential risk factors for the development of POD, preoperative anticholinergic medication had a medium effect ${ }^{41}$ for the development of such condition. Further, age was identified to display a medium effect on the potential development of POD. A comparable effect on the development of POD was identified for the preoperative EuroSCORE. However, such finding needs to be interpreted with caution, as age is one of the parameters utilised for the calculation of the EuroSCORE. A reduced preoperative AChE activity also had a medium effect on the development of POD. These findings both demonstrate the importance of a cholinergic deficit and of age as risk for the development of POD. However, when interpreting these findings in an external framework, other parameters which have not been assessed in the present study may be of importance: most importantly, frailty has a demonstrated high impact on the development of POD. In a recently published meta-analysis the OR of frailty for the development of POD was higher $(\mathrm{OR}>9)$ than any of the parameters studied within this trial. Hence, future studies need to address the importance of the factors identified within this study and other factors such as frailty or cognitive impairment.

Patients with POD had a significantly longer duration of anaesthesia and were also operated on for longer periods
Table 3 Univariate and multivariate analysis of parameters associated with length of stay in the intensive care unit

\begin{tabular}{|c|c|c|c|c|}
\hline & Univariate analysis & & $\begin{array}{l}\text { Multivariate } \\
\text { analysis }\end{array}$ & \\
\hline & Median $(95 \% \mathrm{Cl})$ & $P$ value & HR (95\% Cl) & $P$ value \\
\hline Age & & 0.97 & & \\
\hline$>71$ years & 0.75 (0.65 to 0.86$)$ & & & \\
\hline$<71$ years & 0.79 (0.56 to 1.03$)$ & & & \\
\hline BMI & & 0.24 & & \\
\hline$>27.5$ & 0.79 (0.68 to 0.91$)$ & & & \\
\hline$\leq 27.5$ & 0.71 (0.48 to 0.94$)$ & & & \\
\hline Sex & & 0.89 & & \\
\hline Male & 0.75 (0.55 to 0.95$)$ & & & \\
\hline Female & 0.75 (0.64 to 0.86$)$ & & & \\
\hline EuroSCORE & & $<0.01$ & & 0.33 \\
\hline$\geq 4$ & 0.79 (0.65 to 0.94$)$ & & & \\
\hline$<4$ & 0.42 (0.11 to 0.72$)$ & & & \\
\hline
\end{tabular}

\begin{tabular}{|c|c|c|c|c|}
\hline $\begin{array}{l}\text { Known alcohol } \\
\text { abuse }\end{array}$ & & 0.76 & & \\
\hline Present & 0.75 (0.66 to 0.84$)$ & & & \\
\hline Absent & $0.38(-)^{*}$ & & & \\
\hline $\begin{array}{l}\text { Anticholinergic } \\
\text { premedication }\end{array}$ & & 0.05 & & 0.39 \\
\hline Present & 0.75 (0.59 to 0.91$)$ & & & \\
\hline Absent & 0.75 (0.64 to 0.86$)$ & & & \\
\hline $\begin{array}{l}\text { Length of } \\
\text { ventilation }\end{array}$ & & $<0.01$ & $\begin{array}{l}2.77 \text { (1.83- } \\
4.2)\end{array}$ & $<0.01$ \\
\hline$>456$ min & 1.04 (0.87 to 1.2$)$ & & & \\
\hline$<456 \min$ & 0.33 (0.28 to 0.39$)$ & & & \\
\hline $\begin{array}{l}\text { Transfusion of } \\
\text { PRBC }\end{array}$ & & 0.04 & & 0.98 \\
\hline Present & $0.92(0.76$ to 1.07$)$ & & & \\
\hline Absent & 0.5 (0.28 to 0.72$)$ & & & \\
\hline $\begin{array}{l}\text { PO day } 1 \text { AChE } \\
\text { activity }\end{array}$ & & 0.03 & & 0.47 \\
\hline$<44.3 \mathrm{U} / \mathrm{g} \mathrm{Hb}$ & 0.79 (0.66 to 0.93$)$ & & & \\
\hline$>44.3 \mathrm{U} / \mathrm{g} \mathrm{Hb}$ & 0.71 (0.44 to 0.98$)$ & & & \\
\hline $\begin{array}{l}\mathrm{PO} \text { day } 1 \mathrm{BChE} \\
\text { activity }\end{array}$ & & $<0.01$ & $\begin{array}{l}1.84(1.24- \\
2.75)\end{array}$ & $<0.01$ \\
\hline$<2762 \mathrm{U} / \mathrm{g} \mathrm{Hb}$ & 1 (0.84 to 1.16$)$ & & & \\
\hline$>2762 \mathrm{U} / \mathrm{g} \mathrm{Hb}$ & $0.5(0.29$ to 0.71$)$ & & & \\
\hline Delirium & & $<0.01$ & $\begin{array}{l}1.79(1.1- \\
2.91)\end{array}$ & 0.02 \\
\hline Present & 1.08 (0.48 to 1.69$)$ & & & \\
\hline Absent & 0.71 (0.51 to 0.91 ) & & & \\
\hline
\end{tabular}

Data comparisons were made with Kaplan-Meier estimates for univariate analysis. Column median indicates median of parameter displayed. Coxregression analysis with stepwise exclusion was used for multivariate analysis. For multivariate analysis HR is only displayed in significant outcome parameters/where applicable.

${ }^{*}$ No OR calculated because the median survival time was not reached. AChE, acetylcholinesterase; BChE, butyrylcholinesterase; BMI, body mass index; EuroSCORE, European System for Cardiac Operative Risk Evaluation; PO, postoperative; PRBC, packed red blood cells. 
of time. Long-lasting surgery is associated with many other risk factors such as hypoxaemia, pain and disturbance of the sleep-wake rhythm. ${ }^{392}$ The anaesthesia itself interferes with various neuronal processes in the brain. It interacts with ion channels, such as the nicotinic ACh receptors, neurotransmitters and second messengers, as well as metabolic processes. ${ }^{43}$ The factors mentioned may have influenced the development of POD.

The effects of a POD are far-reaching. In our study, patients with POD not only stayed longer in the ICU, they also spent significantly more days in hospital postoperatively. These observations may be attributed to multiple factors such as delayed mobilisation and physiotherapy. ${ }^{44}$ Patients with POD require more intensive care from nurses and physicians, so that a transfer to the normal ward is only possible with delay and resulting in higher costs. ${ }^{45}$ In a study published in 2004, Ely et al showed that delirium is an independent predictor of significantly higher 6-month mortality and prolonged hospitalisation in ventilated patients in the ICU. ${ }^{46}$ Our patients did not show an increased in-hospital mortality in patients with POD while we, however, did not follow-up patients for 6 months. Conclusions on associations between long-term mortality and cholinesterase activity may therefore not be drawn from the results of our study.

To determine the diagnosis of delirium, the CAM-ICU was used, which is recommended by clinical guidelines. ${ }^{47}$ While the CAM-ICU test is a tool for the diagnosis of delirium with the benefits of rapid assessment and no requirement for verbal communication with the patient, the CAM-ICU test does not provide information about motor subtypes of delirium. ${ }^{48}$ We believe that future studies addressing this question are potentially of value to help understanding the pathology of this disease.

\section{Strengths and limitations}

Our study has several limitations that must be considered when evaluating the results. This study comprises exclusively cardiac surgery patients. Whether these data can be extrapolated to other patient cohorts remains unclear and warrants further validation. On a statistical note, we have not performed multiple comparison for the assessment of enzyme activities with a consecutive potential increase of the alpha error.

While the literature proposes a myriad of risk factors for the development of POD, differences in the methodology based on different definitions of delirium, differences in assessment of both risk factors and delirium and others, do not allow for a definitive list of risk factors. In conclusion, confounding by potential risk factors not addressed within this study (eg, frailty or cognitive impairment) may limit the application of the results found within this study. ${ }^{1011}$

One limitation may be found in the lack of a consensus on a single classification system for anticholinergic medication. While several classification systems exist (as reviewed by Durán et al), ${ }^{49}$ the true effects of preoperative anticholinergic medication may differ depending on the classification system applied for analysis.

It is known that delirium can fluctuate strongly and occur acutely during the course of the day. ${ }^{50}$ In this study, only one measurement was performed in the morning of the day of measurement. Thus, it is possible that not all patients who developed delirium were detected with the applied screening method. One limitation of our study might be the short duration of 2 days measurement, which might have led to patients with POD not being diagnosed with delirium. Further, a substantial variation of results was observed within the study, potentially limiting the conclusions drawn from the results.

The patient population was reduced from a total of 150 patients to 114 , who were ultimately included for analysis. One reason for the exclusion of patients was excessive sedation at postoperative days 1 and 2 and thus an exclusion criterion for the CAM-ICU. Future studies should cover a longer observation period in order to be able to include such patients for analysis and to enable further conclusions to be drawn about the temporal development of POD.

\section{CONCLUSIONS}

We demonstrated that the development of POD after cardiac surgery correlates with postoperative decrease of BChE activity. In addition, patients who developed POD in the course of surgery showed significantly lower preoperative AChE activity as compared with patients without POD. We were able to identify a low preoperative AChE activity, an anticholinergic premedication, an increased EuroSCORE and a higher age as predictors for development of POD. In addition, patients with POD differed from their peers by a longer postoperative ventilation time, an extended stay at the ICU and prolonged hospitalisation.

Our data show that the cholinergic deficit hypothesis may be of importance for the development of POD. Anticholinergic medication may intervene in this pathophysiological system and may influence AChE and BChE activity resulting in neuroinflammation.

There are various studies investigating the risk factors for the occurrence of POD. Some correlations in the development of POD have been identified. However, the molecular basis of multifactorial POD has not yet been sufficiently understood. Nonetheless, this is necessary in order to develop preventive measures. Further studies are needed to investigate the exact pathomechanisms of risk factors for such disease.

Contributors EHA: wrote the manuscript, analysed and interpreted the data. VH: conceived the study idea and collected data. SL: collected data, provided critical feedback and contributed to the final version of the manuscript. KZ: supervised the project and contributed to the final version of the manuscript. BS: conceived the study idea, analysed the data and contributed to the final version of the manuscript. All authors read and approved the final version of the manuscript. 
Funding The authors have not declared a specific grant for this research from any funding agency in the public, commercial or not-for-profit sectors.

Competing interests None declared.

Patient consent for publication Not required

Ethics approval The institutional review board approved the conduct of the study prior to its initiation (428/12 of 19 December 2012).

Provenance and peer review Not commissioned; externally peer reviewed.

Data availability statement Data are available upon reasonable request.

Open access This is an open access article distributed in accordance with the Creative Commons Attribution Non Commercial (CC BY-NC 4.0) license, which permits others to distribute, remix, adapt, build upon this work non-commercially, and license their derivative works on different terms, provided the original work is properly cited, appropriate credit is given, any changes made indicated, and the use is non-commercial. See: http://creativecommons.org/licenses/by-nc/4.0/.

\section{ORCID iD}

Elisabeth Hannah Adam http://orcid.org/0000-0001-5008-6293

\section{REFERENCES}

1 European Delirium Association, American Delirium Society. The DSM-5 criteria, level of arousal and delirium diagnosis: inclusiveness is safer. BMC Med 2014:12:141-41.

2 Grover S, Avasthi A. Clinical practice guidelines for management of delirium in elderly. Indian J Psychiatry 2018;60:S329-40.

3 Leentjens AFG, Rundell J, Rummans T, et al. Delirium: an evidencebased medicine (EBM) monograph for psychosomatic medicine practice, comissioned by the Academy of psychosomatic medicine (APM) and the European association of consultation liaison psychiatry and psychosomatics (EACLPP). J Psychosom Res 2012;73:149-52.

4 Kazmierski J, Kowman M, Banach M, et al. Incidence and predictors of delirium after cardiac surgery: results from the IPDACS study. $J$ Psychosom Res 2010;69:179-85.

5 Rudolph JL, Jones RN, Levkoff SE, et al. Derivation and validation of a preoperative prediction rule for delirium after cardiac surgery. Circulation 2009;119:229-36.

6 Koster S, Oosterveld FGJ, Hensens AG, et al. Delirium after cardiac surgery and predictive validity of a risk checklist. Ann Thorac Surg 2008;86:1883-7.

7 Schimmer C, Reents W, Berneder S, et al. Prevention of sternal dehiscence and infection in high-risk patients: a prospective randomized multicenter trial. Ann Thorac Surg 2008;86:1897-904.

8 Koster S, Hensens AG, Schuurmans MJ, et al. Consequences of delirium after cardiac operations. Ann Thorac Surg 2012;93:705-11.

9 Smulter N, Lingehall HC, Gustafson Y, et al. Delirium after cardiac surgery: incidence and risk factors. Interact Cardiovasc Thorac Surg 2013;17:790-6.

10 Persico I, Cesari M, Morandi A, et al. Frailty and delirium in older adults: a systematic review and meta-analysis of the literature. J Am Geriatr Soc 2018;66:2022-30.

11 Aitken SJ, Blyth FM, Naganathan V. Incidence, prognostic factors and impact of postoperative delirium after major vascular surgery: a meta-analysis and systematic review. Vasc Med 2017;22:387-97.

12 Inouye SK. The dilemma of delirium: clinical and research controversies regarding diagnosis and evaluation of delirium in hospitalized elderly medical patients. Am J Med 1994;97:278-88.

13 Rothenhäusler H-B, Grieser B, Nollert G, et al. Psychiatric and psychosocial outcome of cardiac surgery with cardiopulmonary bypass: a prospective 12-month follow-up study. Gen Hosp Psychiatry 2005;27:18-28.

14 Rudolph JL, Marcantonio ER, Culley DJ, et al. Delirium is associated with early postoperative cognitive dysfunction. Anaesthesia 2008;63:941-7.

15 Trzepacz P, van der Mast R, Lindesay J, et al. Delirium in old age 2002.

16 Downes GB, Granato M. Acetylcholinesterase function is dispensable for sensory neurite growth but is critical for neuromuscular synapse stability. Dev Biol 2004;270:232-45.

17 Plaschke K, Hauth S, Jansen C, et al. The influence of preoperative serum anticholinergic activity and other risk factors for the development of postoperative cognitive dysfunction after cardiac surgery. J Thorac Cardiovasc Surg 2013;145:805-11.

18 Gamberini M, Bolliger D, Lurati Buse GA, et al. Rivastigmine for the prevention of postoperative delirium in elderly patients undergoing elective cardiac surgery--a randomized controlled trial. Crit Care Med 2009;37:1762-8.

19 Das UN. Acetylcholinesterase and butyrylcholinesterase as markers of low-grade systemic inflammation. Ann Hepatol 2012;11:409-11.

20 Gabriel AJ, Almeida MR, Ribeiro $\mathrm{MH}$, et al. Influence of butyrylcholinesterase in progression of mild cognitive impairment to Alzheimer's disease. Journal of Alzheimer's Disease 2018;61:1097-105

21 Greig NH, Utsuki T, Ingram DK, et al. Selective butyrylcholinesterase inhibition elevates brain acetylcholine, augments learning and lowers Alzheimer beta-amyloid peptide in rodent. Proc Natl Acad Sci U S A 2005;102:17213-8.

22 Cerejeira J, Batista P, Nogueira V, et al. Low preoperative plasma cholinesterase activity as a risk marker of postoperative delirium in elderly patients. Age Ageing 2011;40:621-6.

23 John M, Ely EW, Halfkann D, et al. Acetylcholinesterase and butyrylcholinesterase in cardiosurgical patients with postoperative delirium. $j$ intensive care 2017:5:29.

24 Müller A, Olbert M, Heymann A, et al. Relevance of peripheral cholinesterase activity on postoperative delirium in adult surgical patients (CESARO): a prospective observational cohort study. Eur Anaesthesiol 2019;36:114-22.

25 Guenther U, Popp J, Koecher L, et al. Validity and reliability of the CAM-ICU Flowsheet to diagnose delirium in surgical ICU patients. J Crit Care 2010;25:144-51.

26 Schiebeler $\mathrm{H}$, von Mayersbach $\mathrm{H}$. Circadian variations of acetylcholine esterase (E.C.3.1.1.7) in rat brains. Int $J$ Chronobiol 1974;2:281-9.

27 Worek F, Mast U, Kiderlen D, et al. Improved determination of acetylcholinesterase activity in human whole blood. Clinica Chimica Acta 1999;288:73-90.

28 Zimmermann V. CHE-Check technical information. Available: http:// www.securetec.net/sites/default/files/03_Produkte/ChECheck/ Dateien/Schnelltest\%20Bestimmung\%20Cholinesterase ChE check_mobile_Methode_CH1206K_v02_DE.pdf

29 Shihana F, Worek F, Dassanayake GA, et al. Evaluation of the accuracy of "ChE check mobile" in measurement of acetylcholinesterase in pesticide poisoning. Clin Toxicol 2019;57:411-4.

30 Nashef SAM, Roques F, Michel P, et al. European system for cardiac operative risk evaluation (EuroSCORE). European Journal of CardioThoracic Surgery 1999;16:9-13.

31 Ancelin ML, Artero S, Portet F, et al. Non-degenerative mild cognitive impairment in elderly people and use of anticholinergic drugs: longitudinal cohort study. BMJ 2006;332:455-9.

32 Althouse AD. Adjust for Multiple Comparisons? It's Not That Simple. Ann Thorac Surg 2016;101:1644-5.

33 Müller M. Molekular-Dynamik-Simulationen zum KatalyseMechanismus Der acetylcholinesterase 2002

34 Zivkovic AR, Bender J, Brenner T, et al. Reduced butyrylcholinesterase activity is an early indicator of traumainduced acute systemic inflammatory response. J Inflamm Res 2016:9:221-30.

35 Hubbard RE, O'Mahony MS, Calver BL, et al. Plasma esterases and inflammation in ageing and frailty. Eur J Clin Pharmacol 2008:64:895-900.

36 Abou-Hatab Ket al. Relationship between age and plasma esterases. Age Ageing 2001;30:41-5.

37 Lepage L, Schiele F, Gueguen R, et al. Total cholinesterase in plasma: biological variations and reference limits. Clin Chem 1985;31:546-50.

38 Rider JA, Hodges JL, Swader J, et al. Plasma and red cell cholinesterase in 800 healthy blood donors. J Lab Clin Med 1957;50:376-83.

39 Reade MC, Finfer S. Sedation and delirium in the intensive care unit. N Engl J Med 2014;370:444-54.

40 Naja M, Zmudka J, Hannat S, et al. In geriatric patients, delirium symptoms are related to the anticholinergic burden. Geriatr Gerontol Int 2016;16:424-31.

41 Chen $\mathrm{H}$, Cohen $\mathrm{P}$, Chen $\mathrm{S}$. How big is a big odds ratio? interpreting the magnitudes of odds ratios in epidemiological studies. Commun Stat Simul Comput 2010;39:860-4.

42 Figueroa-Ramos MI, Arroyo-Novoa CM, Lee KA, et al. Sleep and delirium in ICU patients: a review of mechanisms and manifestations. Intensive Care Med 2009;35:781-95.

43 Franks NP, Lieb WR. Molecular and cellular mechanisms of general anaesthesia. Nature 1994;367:607-14.

44 Epstein N. A review article on the benefits of early mobilization following spinal surgery and other medical/surgical procedures. Surg Neurol Int 2014;5:66-73

45 Fruhwald T, Weissenberger-Leduc M, Jagsch $C$, et al. Delirium: an interdisciplinary challenge]. Z Gerontol Geriatr 2014;47:425-38. 
46 Ely EWet al. Delirium as a predictor of mortality in mechanically ventilated patients in the intensive care unit. JAMA 2004;291:1753-62.

47 Barr J, Fraser GL, Puntillo K, et al. Clinical practice guidelines for the management of pain, agitation, and delirium in adult patients in the intensive care unit. Crit Care Med 2013;41:263-306.

48 Miranda F, Arevalo-Rodriguez I, Díaz G, et al. Confusion assessment method for the intensive care unit (CAM-ICU) for the diagnosis of delirium in adults in critical care settings. Cochrane Database of Systematic Reviews 2018;41.

49 Durán CE, Azermai M, Vander Stichele RH. Systematic review of anticholinergic risk scales in older adults. Eur J Clin Pharmacol 2013;69:1485-96.

50 Theuerkauf N, Guenther U. [Delirium on the ICU: clinical impact, diagnostic workup, and therapy]. Med Klin Intensivmed Notfmed 2014;109:129-36. 